\title{
GENERATING THE FULL TRANSFORMATION SEMIGROUP USING ORDER PRESERVING MAPPINGS
}

\author{
P. M. HIGGINS \\ Department of Mathematics, University of Essex, Wivenhoe Park, Colchester, CO4 3SQ, United Kingdom \\ e-mail:peteh@essex.ac.uk
}

\author{
J. D. MITCHELL and N. RUŠKUUC \\ Mathematical Institute, North Haugh, St Andrews, Fife, KY16 9SS, United Kingdom \\ e-mail:nr1@st-and.ac.uk
}

(Received 8 October, 2002; accepted 2 February, 2003)

\begin{abstract}
For a linearly ordered set $X$ we consider the relative rank of the semigroup of all order preserving mappings $\mathcal{O}_{X}$ on $X$ modulo the full transformation semigroup $\mathcal{T}_{X}$. In other words, we ask what is the smallest cardinality of a set $A$ of mappings such that $\left\langle\mathcal{O}_{X} \cup A\right\rangle=\mathcal{T}_{X}$. When $X$ is countably infinite or well-ordered (of arbitrary cardinality) we show that this number is one, while when $X=\mathbb{R}$ (the set of real numbers) it is uncountable.
\end{abstract}

2000 Mathematics Subject Classification. 20M20, 06A05.

1. Introduction. For a semigroup $S$, the 'classical' idea of rank is concerned with finding minimum size generating sets for $S$; see [6] or [10]. When working with a finitely generated semigroup $S$ determining the $\operatorname{rank}$ of $S$, denoted $\operatorname{rank}(S)$, is a natural consideration. However, for an uncountable semigroup $S$ the rank of $S$ is $|S|$, and so the classical notion of rank provides us with no information. We introduce a different rank property which allows us to 'measure', from a certain perspective, a given semigroup with respect to some distinguished subsemigroups. For a semigroup $S$, if $A \subseteq S$ then we call the minimum cardinality of a set $B$ such that

$$
\langle A \cup B\rangle=S,
$$

the relative rank of $S$ modulo $A$. Alternatively, we may refer to this cardinality as the relative rank of $A$ in $S$; which we denote by $\operatorname{rank}(S: A)$. This subject has been studied in the context of groups in [2] and [14]. In these papers, so called large subgroups of the symmetric group $\mathcal{S}_{X}$, over an infinite set $X$, were considered. The relative rank of the full transformation semigroup $\mathcal{T}_{X}$, over an infinite set $X$, modulo various standard subsemigroups was first considered in [7]. It was shown in [11] that the relative rank of $\mathcal{T}_{X}$ modulo $\mathcal{S}_{X}$ is two. In the same paper it was shown that the relative rank of the set of all idempotent maps on $X$ in $\mathcal{T}_{X}$ is, also, two. Sierpiński [15] showed that any countable set of maps from $X$ to $X$ is contained in a 2-generated subsemigroup of $\mathcal{T}_{X}$. An alternative proof of this was given by Banach [1]; see also [8]. An immediate corollary of this result is that the relative rank of a subset of $\mathcal{T}_{X}$ is either uncountable or at most two. The corresponding result, that any countable set of permutations is contained in a 2-generated subgroup of $\mathcal{S}_{X}$, was given some years later in [3]. The 
analogues of these results in the semigroup of all binary relations, the semigroup of all partial maps, and the symmetric inverse semigroup were proven in [8].

In this paper we consider the relative rank of the full transformation semigroup $\mathcal{T}_{X}$, where $(X, \leq)$ is an infinite linearly ordered set, modulo the subsemigroup $\mathcal{O}_{X}$ of all order preserving maps on $X$. Recall, that a map $\alpha \in \mathcal{T}_{X}$ is order preserving if

$$
x \leq y \text { implies } x \alpha \leq y \alpha,
$$

for all $x, y \in X$.

For finite $X$ (of size $n$, say) the semigroup $\mathcal{O}_{X}$ has been studied extensively. Its order is $\left(\begin{array}{c}2 n-1 \\ n-1\end{array}\right)$, its rank, in the classical sense, is $n$, it is idempotent generated and its idempotent rank is $2 n-2$ (see [4] and [9]). Furthermore, it is easy to see that

$$
\operatorname{rank}\left(\mathcal{T}_{X}: \mathcal{O}_{X}\right)=2
$$

Indeed, it is well-known that it is possible to generate $\mathcal{T}_{X}$ using elements of $\mathcal{S}_{X}$ and an arbitrary map $\alpha$ with the property that $|\operatorname{im}(\alpha)|=n-1$. If we choose $\alpha \in \mathcal{O}_{X}$ then the result follows from the observation that $\mathcal{O}_{X} \cap \mathcal{S}_{X}=\left\{1_{X}\right\}$ and the fact that $\operatorname{rank}\left(\mathcal{S}_{X}\right)=2$.

In $[8]$ the case where $X=\mathbb{N}$ (with the usual ordering) was considered, and it was shown that

$$
\operatorname{rank}\left(\mathcal{T}_{\mathbb{N}}: \mathcal{O}_{\mathbb{N}}\right)=1
$$

In this paper we build on the above example and show that

$$
\operatorname{rank}\left(\mathcal{T}_{X}: \mathcal{O}_{X}\right)=1,
$$

when $X$ is an arbitrary countable linearly ordered set or an arbitrary well-ordered set (of any cardinality) but that $\operatorname{rank}\left(\mathcal{T}_{X}: \mathcal{O}_{X}\right)$ can be uncountable for some (uncountable, non-well-ordered) linearly ordered sets $X$.

2. Countable linearly ordered sets. In this section we prove the following result.

THEOREM 2.1. Let $X$ be a countable linearly ordered set. The relative rank of $\mathcal{T}_{X}$ modulo $\mathcal{O}_{X}$ is one.

For the remainder of this section $X$ will be a fixed countably infinite linearly ordered set. For $x, y \in X$ with $x<y$ we define

$$
\begin{aligned}
& {[x, y]=\{z \in X: x \leq z \leq y\},(x, y)=\{z \in X: x<z<y\},} \\
& (x, y]=\{z \in X: x<z \leq y\},[x, y)=\{z \in X: x \leq z<y\} .
\end{aligned}
$$

We call these sets intervals, delimited by $x$ and $y$. An element $d \in X$ is called discrete if there exist $x, y \in X$ with $d \in(x, y)$ such that $(x, d)=(d, y)=\emptyset$. We call an element $r \in X$ right isolated if for every $x \in X$ such that $x<r$ we have $(x, r) \neq \varnothing$ but there exists $y \in X$ with $y>r$ such that $(r, y)=\emptyset$. Note that, in fact, if $r$ is right isolated, then $|(x, r)|=|X|$ for all $x<r$. Perhaps a better name for an element with the above properties is a right isolated left limit point, but for the sake of convenience we shall use the shorter name. Left isolated elements are defined analogously. Note that if $X$ has a smallest or largest element then this element is not right or left isolated or discrete with 
the current definitions. To remedy this, if $x_{0} \in X$ is the smallest element of $X$ and there exists $y \in X$ such that $y \neq x_{0}$ and $\left(x_{0}, y\right)=\emptyset$ then we shall call $x_{0}$ discrete; otherwise we call $x_{0}$ left isolated. Analogously, if $X$ has a largest element then it is either discrete or right isolated. Finally, an element $t \in X$ (which is neither the largest nor the smallest element of $X)$ is called a limit point if $(x, t) \neq \emptyset$ and $(t, y) \neq \emptyset$ for every $x<t$ and for every $y>t$.

We now start a sequence of lemmas, leading to the proof of Theorem 2.1. Throughout we take $\mathbb{N}=\{1,2, \ldots\}$.

LEMMA 2.2. Let $X$ be a countable linearly ordered set consisting entirely of limit points, and let $\lambda$ be any function from $X$ to $\mathbb{N}$. Then there exists an order preserving injection $\alpha$ from $X$ to $X$ such that $|X \backslash \operatorname{im}(\alpha)|=|X|(=|\operatorname{im}(\alpha)|)$ satisfying $x \alpha \lambda \geq x \lambda$, for all $x \in X$.

Proof. We start by finding an interval $I \subseteq X$ such that for every $x, y, z \in I$, with $x<y$, there exists $t \in(x, y)$ such that $t \lambda \geq z \lambda$. Let $J$ be an arbitrary interval. One of the following alternatives holds:

(i) for every subinterval of $J$ delimited by $x, y \in J$, with $x<y$, and for every $n \in \mathbb{N}$ there exists $z \in(x, y)$ such that $z \lambda>n$; or

(ii) there exists a subinterval $K \subseteq J$ and there exists $n \in \mathbb{N}$ such that $x \lambda \leq n$, for each $x \in K$.

If condition (i) holds then the interval $J$ has the required property and we let $I=J$. Assume that condition (ii) holds. If for all $x, y \in K$, with $x<y$, there exists $z \in(x, y)$ such that $z \lambda=n$, then $K$ satisfies the necessary condition and we let $I=K$. Otherwise, there exists a subinterval $K_{1} \subseteq K$ such that $x \lambda \leq n-1$, for all $x \in K_{1}$. We consider $K_{1}$ in the same way as we have just considered $K$, so that if for all $x, y \in K_{1}$, with $x<y$, there exists $z \in(x, y)$ such that $z \lambda=n-1$ then we let $I=K_{1}$. Otherwise, there exists a subinterval $K_{2} \subseteq K_{1}$ such that $x \lambda \leq n-2$, for all $x \in K_{2}$. We repeat this process to give the sequence of non-empty subintervals:

$$
K=K_{0} \supseteq K_{1} \supseteq K_{2} \supseteq K_{3} \supseteq \cdots,
$$

where $x \lambda \leq n-i$ for every $x \in K_{i}$. Note that this process always terminates with $i=$ $n-1$ at latest.

Let $(a, b) \subseteq I$ be an arbitrary interval. Note that since $a$ and $b$ are both limit points we have $|(a, b)|=|X|$. We define $\alpha \in \mathcal{O}_{X}$ inductively as follows. First we define $\alpha$ on the points $a$ and $b$, so that $b \alpha=b$ and $a \alpha=c$, where $c \in(a, b)$ with $c \lambda \geq a \lambda$. Such a point $c$ exists from the defining property of $I$.

Next, we enumerate the elements of $[a, b]$ :

$$
b=e_{0}, a=e_{1}, e_{2}, e_{3}, \ldots
$$

For $k \in \mathbb{N}$, our inductive hypothesis is that the elements $e_{0} \alpha, e_{1} \alpha, e_{2} \alpha, \ldots, e_{k} \alpha \in[c, b]$ are defined so that $\alpha$ is injective, order preserving and satisfies $e_{i} \alpha \lambda \geq e_{i} \lambda$ for every $i \in\{0,1, \ldots, k\}$. To define $e_{k+1} \alpha$, we find the largest $e_{i} \in[a, b]$ with $e_{i}<e_{k+1}$, where $i \leq k$, and the smallest $e_{j} \in[a, b]$ with $e_{k+1}<e_{j}$, where $j \leq k$. Since $i, j \leq k$ the elements $e_{i} \alpha$ and $e_{j} \alpha$ are already defined and $e_{i} \alpha<e_{j} \alpha$. From the definition of $I$ there exists $y \in\left(e_{i} \alpha, e_{j} \alpha\right)$ such that $y \lambda \geq e_{k+1} \lambda$ and so we define $e_{k+1} \alpha=y$. Finally, for $x<a$ and for $x>b$ we let $x \alpha=x$. Note that since $a$ is a limit point and $[a, c) \cap \operatorname{im}(\alpha)=\emptyset$ we have $|X \backslash \operatorname{im}(\alpha)|=|X|$. 
We use the above lemma to prove a more general result for linearly ordered countably infinite sets which contain elements that are not limit points.

LEMMA 2.3. Let $X$ be a countable linearly ordered set. Then there exists an order preserving injection $\alpha$ from $X$ to $X$ such that $|X \backslash \operatorname{im}(\alpha)|=|X|(=|\operatorname{im}(\alpha)|)$.

Proof. There are two cases to consider.

Case 1. There is an infinite sequence of consecutive discrete points in $X$. It is clear that any such sequence is either strictly increasing or strictly decreasing. Without loss of generality, we assume that $\left\{y_{1}<y_{2}<\cdots\right\} \subseteq X$ is an increasing sequence of consecutive discrete points. Define $\alpha$ from $X$ to $X$ by

$$
x \alpha= \begin{cases}y_{2 i} & x=y_{i}(i \in \mathbb{N}) \\ x & x \notin\left\{y_{1}, y_{2}, \ldots\right\} .\end{cases}
$$

The map $\alpha$ is an order preserving injection and $X \backslash \operatorname{im}(\alpha)=\left\{y_{1}, y_{3}, \ldots\right\}$.

Case 2. No infinite sequence of consecutive discrete points exists. Without loss of generality assume that $X$ has no smallest or largest point. (Indeed, if $X$ had a smallest point, say, then it would start as $x_{1}<x_{2}<\cdots<x_{k}<l$, where each $x_{i}$ is a discrete point and $l$ is a left isolated point. But then we can consider $X \backslash\left\{x_{1}, \ldots, x_{k}, l\right\}$.)

Consider an arbitrary right isolated point $r$. Then there exists $x_{1}$ such that $r<x_{1}$ and $\left(r, x_{1}\right)=\emptyset$. Clearly, $x_{1}$ is either a discrete point or a left isolated point. If $x_{1}$ is discrete then there exists $x_{2}>x_{1}$ such that $\left(x_{1}, x_{2}\right)=\emptyset$. By assumption we can continue this for only finitely many steps to obtain a finite sequence $r, x_{1}, x_{2}, \ldots, x_{k}, l$ where each $x_{i}$ is a discrete point and $l$ is left isolated. Thus for every right isolated $r \in X$ there exists a corresponding left isolated $l \in X$ such that the interval $(r, l)$ is finite. Let $\rho$ be the equivalence relation with equivalence classes $\{t\}$, where $t$ is a limit point, and $\left\{r, x_{1}, x_{2}, \ldots, x_{k}, l\right\}$ where $x_{i}$ is a discrete point for each $i \in\{1,2, \ldots, k\}, l$ is left isolated and $r$ is right isolated. Let $\bar{X}=X / \rho$, the quotient of $X$ by $\rho$. The order on $X$ induces a (linear) order on $\bar{X}$ :

$$
x / \rho \leq y / \rho \text { if and only if } x / \rho=y / \rho \text { or } x^{\prime}<y^{\prime} \text { for all } x^{\prime} \in x / \rho, y^{\prime} \in y / \rho .
$$

We claim that every point $x / \rho$ of $\bar{X}$ is a limit point. We have two cases to consider, when $x / \rho=\{x\}$ and when $x / \rho=\left\{r, x_{1}, x_{2}, \ldots, x_{k}, l\right\}$, for some $k \geq 0$.

In the first case, we have that $x$ is a limit point in $X$. Let $y \in X$ with $y / \rho<x / \rho$. We show that $(y / \rho, x / \rho) \neq \emptyset$. From the definition of the order on $\bar{X}$ we have $y<x$ and so $|(y, x)|=|X|$. It follows that there exists $z \in(y, x)$ such that $z \neq x$ and $z \notin y / \rho$, since $y / \rho$ is finite. Since $x / \rho$ is a singleton this implies that $z / \rho \in(y / \rho, x / \rho)$ and so $(y / \rho, x / \rho) \neq \emptyset$, as required. An analogous argument shows that for any $z \in X$ with $x / \rho<z / \rho$ we have $(x / \rho, z / \rho) \neq \emptyset$. It follows that $x / \rho$ is a limit point.

In the second case, note that for any $y, z \in X$ such that $y / \rho<x / \rho<z / \rho$ we have $y<r<l<z$. Since $r$ is a right isolated point it follows that $(y, r)$ is infinite. Again, since $y / \rho$ and $r / \rho$ are finite there exists $t \in(y, r)$ such that $t \notin y / \rho$ and $t \notin r / \rho$. This implies that $t / \rho \in(y / \rho, r / \rho)=(y / \rho, x / \rho)$ and so $(y / \rho, x / \rho) \neq \emptyset$, as required. An analogous argument shows that $(x / \rho, z / \rho) \neq \emptyset$. It follows that $x / \rho$ is a limit point.

We now label each element of $\bar{X}$ according to the size of its class, so that we may apply Lemma 2.2. More precisely, we define $\lambda: \bar{X} \rightarrow \mathbb{N}$ by

$$
(x / \rho) \lambda=|x / \rho| .
$$


By Lemma 2.2 there exists an order preserving bijection $\bar{\alpha}$ from $\bar{X}$ to $\bar{X}$ satisfying

$$
(x / \rho) \bar{\alpha} \lambda \geq(x / \rho) \lambda,
$$

such that $\bar{X} \backslash \operatorname{im}(\bar{\alpha})$ is infinite. We shall now 'lift' the function $\bar{\alpha}$ to a function $\alpha: X \rightarrow X$ as follows:

$$
x \alpha= \begin{cases}y & \text { if } x \text { is a limit point and }(x / \rho) \bar{\alpha}=y / \rho=\{y\} \text { where } y \text { is a limit point } \\ r & \text { if } x \text { is a limit point and }(x / \rho) \bar{\alpha}=\left\{r, x_{1}, x_{2}, \ldots, x_{k}, l\right\} \\ x_{i}^{\prime} & \text { if } x=x_{i} \text { in }\left\{r=x_{0}, x_{1}, \ldots, x_{k}, x_{k+1}=l\right\} \in \bar{X} \text { and } \\ & (x / \rho) \bar{\alpha}=\left\{r^{\prime}=x_{0}^{\prime}, x_{1}^{\prime}, \ldots, x_{s}^{\prime}, x_{s+1}^{\prime}=l^{\prime}\right\} \text { for } s \geq k\end{cases}
$$

In the final case, since $\bar{\alpha}$ satisfies $(x / \rho) \bar{\alpha} \lambda=|(x / \rho) \bar{\alpha}| \geq|x / \rho|=(x / \rho) \lambda$, it is clear that, under $\bar{\alpha}$, the image of $x / \rho=\left\{r=x_{0}, x_{1}, \ldots, x_{k}, x_{k+1}=l\right\}$ must be a set with at least $k+2$ elements. Note that, $x \alpha \in(x / \rho) \bar{\alpha}$ for every $x \in X$.

For arbitrary $x, y \in X$, with $x<y$, we show that $x \alpha<y \alpha$ and hence $\alpha$ is order preserving and injective. There are two cases to consider. Firstly, if $x / \rho \neq y / \rho$ then $(x / \rho) \bar{\alpha}<(y / \rho) \bar{\alpha}$, since $\bar{\alpha}$ is order preserving and injective. It follows from the definition of the order on $\bar{X}$ that $z<t$, for all $z \in(x / \rho) \bar{\alpha}$ and for all $t \in(y / \rho) \bar{\alpha}$, and hence $x \alpha<y \alpha$. Secondly, if

$$
x / \rho=\left\{r=x_{0}, x_{1}, \ldots, x_{k}, x_{k+1}=l\right\}=y / \rho
$$

then $x=x_{i}$ and $y=x_{j}$ for $i<j$. By definition we have

$$
(x / \rho) \bar{\alpha}=\left\{r^{\prime}=x_{0}^{\prime}, x_{1}^{\prime}, \ldots, x_{s}^{\prime}, x_{s+1}^{\prime}=l^{\prime}\right\}=(y / \rho) \bar{\alpha},
$$

where $s \geq k$ and $x \alpha=x_{i}^{\prime}<x_{j}^{\prime}=y \alpha$, as required. Note that $\operatorname{im}(\alpha) \subseteq \bigcup \operatorname{im}(\bar{\alpha})$, and since $\bar{X} \backslash \operatorname{im}(\bar{\alpha})$ is infinite, it follows that $X \backslash \operatorname{im}(\alpha)$ is infinite.

The next two lemmas allow us to use methods similar to those in the proof of $[\mathbf{8}$, Example 1.6] to encode an arbitrary map into an order preserving map.

LEMMA 2.4. Let $Y$ be a countably infinite linearly ordered set. Then there exists $Z \subseteq Y$ such that either $Z \cong \mathbb{Z}^{+}$or $Z \cong \mathbb{Z}^{-}$

Proof. There are two cases to consider.

Case 1. All the points in $Y$ are discrete points. We construct $Z$ as follows. Let $z_{1} \in Y$ be arbitrary. Then at least one of the sets $\left\{y \in Y: y>z_{1}\right\}$ or $\left\{y \in Y: y<z_{1}\right\}$ is infinite. Without loss of generality we assume that

$$
\left|\left\{y \in Y: y>z_{1}\right\}\right|=|Y| .
$$

We may choose $z_{2}, z_{3}, \ldots$ so that $\left(z_{1}, z_{2}\right)=\emptyset,\left(z_{2}, z_{3}\right)=\emptyset$, etc. Then $Z=$ $\left\{z_{1}, z_{2}, z_{3}, \ldots\right\} \cong \mathbb{Z}^{+}$.

Case 2. There exists a left isolated point, a right isolated point or a limit point in $Y$. Without loss of generality, we assume that there is a right isolated point $r \in Y$. Let $z_{1}<r$ be arbitrary, since $r$ is right isolated we have $\left(z_{1}, r\right) \neq \emptyset$. Hence we may choose $z_{2} \in\left(z_{1}, r\right)$. Continue to choose $z_{3} \in\left(z_{2}, r\right), z_{4} \in\left(z_{3}, r\right)$, etc. Then $Z=\left\{z_{1}, z_{2}, z_{3}, \ldots\right\} \cong$ $\mathbb{Z}^{+}$. 
LEMMA 2.5. Let $X$ be a countable linearly ordered infinite set. Let $Z \subseteq X$ be such that $Z \cong \mathbb{Z}^{+}$or $Z \cong \mathbb{Z}^{-}$, and let $\alpha$ be an order preserving map from $Z$ to $Z$. Then there exists $\beta \in \mathcal{O}_{X}$ such that $\beta\left\lceil_{Z}=\alpha\right.$.

Proof. We assume, without loss of generality, that $Z=\left\{z_{1}<z_{2}<\cdots\right\} \cong \mathbb{Z}^{+}$and let $\alpha$ be an order preserving map from $Z$ to $Z$. We define $\beta \in \mathcal{T}_{X}$ by

$$
x \beta= \begin{cases}x & \text { if } x<z_{1}, \\ z_{i} \alpha & \text { if } x \in\left[z_{i}, z_{i+1}\right), \\ x & \text { if } x>z_{i}, \text { for every } i \in \mathbb{N} .\end{cases}
$$

It is easy to verify that $\beta$ is order preserving and $\beta\left\lceil_{z}=\alpha\right.$.

We are now in a position to prove the main result of this section.

Proof of Theorem 2.1. Let $Y \subseteq X$ such that $|Y|=|X \backslash Y|=|X|$ and let $\beta$ be an order preserving bijection from $X$ to $X \backslash Y$; these exist by Lemma 2.3. Assume, without loss of generality, that there exists $Z \subseteq Y$ such that $Z \cong \mathbb{Z}^{+}$and let $Z=\left\{z_{1}<z_{2}<\cdots\right\}$, as described in Lemma 2.4. Since $X \backslash Y$ and $Z$ have the same cardinality there exists a bijection $\epsilon$ from $X \backslash Y$ to $Z$. Note that $\beta \epsilon$ is a bijection from $X$ to $Z$. Let $\delta$ be any mapping from $Z$ to $X$ such that

$$
z_{p_{k}^{j}} \delta=z_{k} \epsilon^{-1} \beta^{-1}
$$

where $p_{k}$ is the $k$ th prime and $j \in \mathbb{N}$ arbitrary. Let $\Delta \in \mathcal{T}_{X}$ be any mapping such that

$$
x \Delta= \begin{cases}x \epsilon & \text { if } x \in X \backslash Y \\ x \delta & \text { if } x \in Z \\ \text { arbitrary } & \text { if } x \in Y \backslash Z\end{cases}
$$

Let $\alpha \in \mathcal{T}_{X}$ be arbitrary. We show that $\alpha$ can be generated using $\Delta$ and elements of $\mathcal{O}_{X}$. We define $\gamma$ from $Z$ to $Z$ inductively. First we define $\gamma$ on $z_{1}$, so that if $z_{1} \epsilon^{-1} \beta^{-1} \alpha \beta \epsilon=z_{k}$ then $z_{1} \gamma=z_{p_{k}}$. For $t>1$ we assume that $\gamma$ is defined and order preserving on $z_{1}, \ldots, z_{t-1}$. To define $z_{t} \gamma$ we first let

$$
M=\max \left\{i: z_{i} \in\left\{z_{1}, \ldots, z_{t-1}\right\} \gamma\right\} .
$$

Since $\beta \epsilon$ is a bijection from $X$ to $Z$ there exists $x \in X$ such that

$$
z_{t} \epsilon^{-1} \beta^{-1}=x
$$

and there exists $z_{s} \in Z$ such that $(x \alpha) \beta \epsilon=z_{s}$. We choose $j \in \mathbb{N}$ such that $p_{s}^{j}>M$ and we define $z_{t} \gamma=z_{p_{s}^{j}}$. Our choice of $j$ ensures that $\gamma$ is order preserving. Let $\eta \in \mathcal{T}_{X}$ be an extension of $\gamma$ to an element of $\mathcal{O}_{X}$, as described in Lemma 2.5.

We claim that

$$
\alpha=\beta \Delta \eta \Delta .
$$

Let $x \in X$ be arbitrary. Since $\beta \Delta$ is a bijection (from $X$ to $Z$ ) there exists a unique element $z_{t} \in Z$ such that $x \beta \Delta=x \beta \epsilon=z_{t}$. Analogously, there exists a unique element $z_{s} \in Z$ such that $x \alpha \beta \Delta=x \alpha \beta \epsilon=z_{s}$. Hence

$$
x \beta \Delta \eta \Delta=x \beta \epsilon \eta \Delta=z_{t} \eta \Delta=z_{t} \gamma \Delta=z_{p_{s}^{j}} \Delta=z_{p_{s}^{j}} \delta=z_{s} \epsilon^{-1} \beta^{-1}=x \alpha .
$$

We have shown that $\alpha \in\left\langle\mathcal{O}_{X}, \Delta\right\rangle$ and so $\operatorname{rank}\left(\mathcal{T}_{X}: \mathcal{O}_{X}\right)=1$. 
3. Well-ordered sets. In this section we extend the result of the previous section to well-ordered sets of arbitrary cardinality. Recall that an ordered set $(X, \leq)$ is wellordered if every subset of $X$ contains a least element. We start by introducing some standard results concerning well-ordered sets which we shall use later. For more details see [5], [12] or [13].

For an arbitrary $x \in X$ we call the set $s(x)=\{y \in X: y<x\}$ the initial segment of $x$. It is well-known that for any two well-ordered sets $X$ and $Y$ either $X$ is isomorphic to $Y, X$ is isomorphic to an initial segment of $Y$ or $Y$ is isomorphic to an initial segment of $X$; see for example [12, Theorem 1]. This induces a natural (well) ordering on the class of all well-ordered sets, so that

$X \leq Y$ if and only if $X \cong Y$ or $X$ is isomorphic to an initial segment of $Y$.

A natural reformulation of this result relates a well-ordered set to its subsets.

Proposition 3.6. Each subset of a well-ordered set $X$ is either isomorphic to $X$ or to an initial segment of $X$.

Another useful and natural consequence of the order on the class of all well-ordered sets is:

Proposition 3.7. No well-ordered set is isomorphic to an initial segment of itself.

For a proof see [12, Lemma 2.2].

In light of the previous section a natural question to ask is whether, or not, there exists an uncountable set $X$ for which the relative rank of $\mathcal{T}_{X}$ modulo $\mathcal{O}_{X}$ is countable? The next result answers this question in the affirmative.

LEMMA 3.8. Let $X$ be an arbitrary infinite set and let $\Omega$ denote the least well-ordered set of cardinality $|X|$. Then the relative rank of $\mathcal{T}_{\Omega}$ modulo $\mathcal{O}_{\Omega}$ is one.

Proof. Let $\Omega_{x}(x \in \Omega)$ denote subsets of $\Omega$ such that $\left|\Omega_{x}\right|=|\Omega|$ and $\Omega_{x} \cap \Omega_{y}=\emptyset$ whenever $x \neq y$. Since each $\Omega_{x}$ has cardinality $|X|$ and $\Omega$ is the smallest well-ordered set of cardinality $|X|$, it follows from Proposition 3.6 and Proposition 3.7 that $\Omega_{x} \cong \Omega$, for all $x \in \Omega$. Fix a mapping $\mu \in \mathcal{T}_{\Omega}$ such that

$$
\Omega_{y} \mu=y \quad(y \in \Omega) .
$$

For an arbitrary $\alpha \in \mathcal{T}_{\Omega}$, we define a map $\beta \in \mathcal{O}_{\Omega}$ by transfinite induction as follows. If $x \alpha=y$ then we define $x \beta=z$, where $z \in \Omega_{y}$ and $z>t \beta$ for every $t<x$. Such an element $z$ exists since $\Omega_{y}(\cong \Omega)$ is not isomorphic to an initial segment of $\Omega$, by Proposition 3.7. For an arbitrary $x \in \Omega$, if $x \alpha=y$ then we have

$$
x \beta \mu=z \mu=y=x \alpha,
$$

and so $\alpha \in\left\langle\mathcal{O}_{\Omega}, \mu\right\rangle$. It follows that $\mathcal{T}_{\Omega}=\left\langle\mathcal{O}_{\Omega}, \mu\right\rangle$ and in particular $\operatorname{rank}\left(\mathcal{T}_{\Omega}: \mathcal{O}_{\Omega}\right)=1$, as required.

We use this lemma to prove the main result of this section:

THEOREM 3.9. Let $X$ be an arbitrary well-ordered set. The relative rank of $\mathcal{T}_{X}$ modulo $\mathcal{O}_{X}$ is one.

Proof. Let $T$ be the smallest well-ordered set of cardinality $|X|$. By Proposition 3.6 either there exists an initial segment of $X$ which is isomorphic to $T$ or $X \cong T$. In 
the latter case the result follows by Lemma 3.8. In the former case, let $Y$ denote the initial segment of $X$ isomorphic to $T$ and let $Z=X \backslash Y$. By the same argument as in the proof of Lemma 3.8, we may find pairwise disjoint sets $Y_{1}, Y_{2}, Y_{3} \subseteq Y$ such that $Y=Y_{1} \cup Y_{2} \cup Y_{3}$ and $Y_{i} \cong Y$, for each $i$. By Lemma 3.8 there exists a map $\epsilon \in \mathcal{T}_{Y_{2}}$ such that $\left\langle\mathcal{O}_{Y_{2}}, \epsilon\right\rangle=\mathcal{T}_{Y_{2}}$. We define a map $\sigma: \mathcal{T}_{Y_{2}} \longrightarrow \mathcal{T}_{X}$ such that for $x \in X$ and $\rho \in \mathcal{T}_{Y_{2}}$

$$
(x)(\rho \sigma)= \begin{cases}x & x \in Z, \\ y \rho & x \in Y, \text { where } y=\min \left\{z \in Y_{2}: z \geq x\right\} .\end{cases}
$$

In the second case, note that such an element $y$ always exists since $Y_{2}$ is well-ordered and is not a subset of an initial segment of $Y$. It is easy to see that $\mathcal{O}_{Y_{2}} \sigma \subseteq \mathcal{O}_{X}$. Since $Y \cong Y_{1}$ and $y \leq z$, for every $y \in Y$ and for every $z \in Z$, we may define an injection $\beta \in \mathcal{O}_{X}$ from $X$ to $Y_{1} \cup Z$ such that $Y \beta=Y_{1}$ and $Z \beta=Z$. Since $Y_{1} \cup Z$ has the same cardinality as $Y_{2}$ we may find a bijection $\delta$ from $Y_{1} \cup Z$ to $Y_{2}$. We let $\bar{\mu}$ be any order preserving bijection from $Y_{2}$ to $Y_{3}$ and define $\mu \in \mathcal{T}_{X}$ by

$$
x \mu= \begin{cases}x & x>y, \text { for all } y \in Y_{2} \\ y \bar{\mu} & y=\min \left\{z \in Y_{2}: z \geq x\right\} .\end{cases}
$$

In fact, $\mu$ is order preserving. In order to see this, it is enough to note that $\{x: x>$ $y$ for all $\left.y \in Y_{2}\right\}=Z$ since $Y_{2}$, being isomorphic to $Y$, is not contained in an initial segment of $Y$. From the definition it is obvious that $\mu$ restricted to either $Z$ or $Y=X \backslash Z$ is order preserving. In addition, $Y \mu=Y_{2} \mu=Y_{3} \subseteq Y, Z \mu=Z$ and $y<z$ for all $y \in Y, z \in Z$.

Next, we define a map $\gamma: Y_{3} \longrightarrow X$ by

$$
y \gamma=y \bar{\mu}^{-1} \delta^{-1} \beta^{-1} .
$$

Note that the domains of the maps $\delta, \epsilon$ and $\gamma$ are disjoint and that their union is $X$ itself. Hence we may define a map $\eta \in \mathcal{T}_{X}$ by

$$
x \eta= \begin{cases}x \delta & x \in Y_{1} \cup Z \\ x \epsilon & x \in Y_{2} \\ x \gamma & x \in Y_{3} .\end{cases}
$$

We claim that $\eta$ together with $\mathcal{O}_{X}$ generates $\mathcal{T}_{X}$. Let $\alpha \in \mathcal{T}_{X}$ be arbitrary. First note that for any $\theta \in \mathcal{T}_{Y_{2}}$ there exists $\rho \in\left\langle\mathcal{O}_{Y_{2}} \sigma, \eta\right\rangle \subseteq\left\langle\mathcal{O}_{X}, \eta\right\rangle\left(\subseteq \mathcal{T}_{X}\right)$ such that $\rho \uparrow_{Y_{2}}=\theta$. This follows from $\left.(\zeta \sigma)\right|_{Y_{2}}=\zeta$ for all $\zeta \in \mathcal{T}_{Y_{2}}, \eta \uparrow_{Y_{2}}=\epsilon$ and $\left\langle\mathcal{O}_{Y_{2}}, \epsilon\right\rangle=\mathcal{T}_{Y_{2}}$.

In particular, since $\beta \delta$ is a bijection from $X$ to $Y_{2}$, we may find a map $\rho \in\left\langle\mathcal{O}_{X}, \eta\right\rangle$ such that $\rho \uparrow_{2}=\delta^{-1} \beta^{-1} \alpha \beta \delta$. Then, for an arbitrary $x \in X$, with $x \beta \delta=y \in Y_{2}$ we have

$$
\begin{aligned}
x \beta \eta \rho \mu \eta & =(x \beta \delta) \rho \mu \eta=y \rho \mu \eta=y \delta^{-1} \beta^{-1} \alpha \beta \delta \mu \eta=(x \alpha) \beta \delta \mu \eta \\
& =(x \alpha \beta \delta \mu) \gamma=x \alpha \beta \delta \mu \bar{\mu}^{-1} \delta^{-1} \beta^{-1}=x \alpha,
\end{aligned}
$$

and so $\alpha \in\left\langle\mathcal{O}_{X}, \eta\right\rangle$ and $\mathcal{T}_{X}=\left\langle\mathcal{O}_{X}, \eta\right\rangle$.

4. Concluding remarks. We can extend the main result of the last section to a wider family of linearly ordered sets by means of the following lemma. 
LEMMA 4.10. Let $X$ be an infinite linearly ordered set such that there exists a subset $Y \subseteq X$ with $|Y|=|X|$ and where any order preserving map from $Y$ to $Y$ can be extended to an order preserving map from $X$ to $X$. Then $\operatorname{rank}\left(\mathcal{T}_{Y}: \mathcal{O}_{Y}\right) \leq 2$ implies $\operatorname{rank}\left(\mathcal{T}_{X}: \mathcal{O}_{X}\right) \leq 2$.

Proof. By assumption, there exist $\epsilon^{\prime}, \delta^{\prime} \in \mathcal{T}_{Y}$ such that $\left\langle\mathcal{O}_{Y}, \epsilon^{\prime}, \delta^{\prime}\right\rangle=\mathcal{T}_{Y}$, and for every $\alpha \in \mathcal{O}_{Y}$ there exists $\eta \in \mathcal{O}_{X}$ such that $\eta \uparrow_{Y}=\alpha$. Let $\epsilon, \delta \in \mathcal{T}_{X}$ be any mappings such that $\left.\epsilon\right|_{Y}=\epsilon^{\prime}$ and $\left.\delta\right|_{Y}=\delta^{\prime}$. Let $\beta: X \rightarrow Y$ be any bijection and let $\gamma \in \mathcal{T}_{X}$ be arbitrary. We show that it is possible to generate $\gamma$ using elements of $\mathcal{O}_{X}$ and four other mappings. Since $\left\langle\mathcal{O}_{Y}, \epsilon^{\prime}, \delta^{\prime}\right\rangle=\mathcal{T}_{Y}$ we see that for any map $\alpha \in \mathcal{T}_{Y}$ there exists $\mu \in\left\langle\mathcal{O}_{X}, \epsilon, \delta\right\rangle$ such that $\mu \uparrow_{Y}=\alpha$. In particular, there exists $\mu \in\left\langle\mathcal{O}_{X}, \epsilon, \delta\right\rangle$ such that

$$
\mu \uparrow_{Y}=\beta^{-1} \gamma \beta
$$

Let $v$ be any extension of $\beta^{-1}$ to an element of $\mathcal{T}_{X}$. For an arbitrary $x \in X$ if $x \beta=y$ then

$$
x \beta \mu \nu=y \mu \nu=y \beta^{-1} \gamma \beta \nu=y \beta^{-1} \gamma=x \gamma .
$$

We have shown that $\gamma \in\left\langle\mathcal{O}_{X}, \delta, \epsilon, \beta, \nu\right\rangle$ and so $\mathcal{T}_{X}=\left\langle\mathcal{O}_{X}, \delta, \epsilon, \beta, v\right\rangle$. It follows from $\left[8\right.$, Corollary 1.2] that $\operatorname{rank}\left(\mathcal{T}_{X}: \mathcal{O}_{X}\right) \leq 2$.

COROLlary 4.11. If $X$ is an arbitrary linearly ordered set, such that there exists a well-ordered subset $Y \subseteq X$, with $|Y|=|X|$, then $\operatorname{rank}\left(\mathcal{T}_{X}: \mathcal{O}_{X}\right) \leq 2$.

Proof. Let $\alpha^{\prime}$ be an order preserving map from $Y$ to $Y$. Then $\alpha \in \mathcal{T}_{X}$ defined by

$$
x \alpha= \begin{cases}x & x>y \text { for all } y \in Y \\ y \alpha^{\prime} & y=\min \{z \in Y: z \geq x\},\end{cases}
$$

is an order preserving map from $X$ to $X$. The result follows by Theorem 3.9 and Lemma 4.10.

Having found the relative rank of $\mathcal{T}_{\mathbb{N}}$ modulo $\mathcal{O}_{\mathbb{N}}$ and the relative rank of $\mathcal{T}_{\mathbb{Q}}$ modulo $\mathcal{O}_{\mathbb{Q}}$ a natural question to ask is: what is the relative rank of $\mathcal{T}_{\mathbb{R}}$ modulo $\mathcal{O}_{\mathbb{R}}$ ?

EXAMPLE 4.12. The relative rank of $\mathcal{T}_{\mathbb{R}}$ modulo $\mathcal{O}_{\mathbb{R}}$ is uncountable. We show this by proving that the cardinality of the semigroup of all order preserving mappings on the reals $\mathbb{R}$ is $2^{\aleph_{0}}<2^{2^{\aleph_{0}}}=\left|\mathcal{T}_{\mathbb{R}}\right|$. For an arbitrary $\alpha \in \mathcal{O}_{\mathbb{R}}$ we show that $\alpha$ is discontinuous at only countably many points in $\mathbb{R}$. Let $D=\{x: \alpha$ is discontinuous at $x\}$. For $x \in D$, let $a_{x}=\sup _{t<x}\{t \alpha\}$ and let $b_{x}=\inf _{t>x}\{t \alpha\}$. Next, define $\beta: D \longrightarrow G$, where $G$ is the family of all open subsets of $\mathbb{R}$, by:

$$
x \beta=\left(a_{x}, b_{x}\right) .
$$

Observe that the family $\{x \beta: x \in D\}$ consists of non-empty pairwise disjoint open sets, hence

$$
|\{x \beta: x \in D\}| \leq \aleph_{0} .
$$

The map $\beta$ is injective and so

$$
|D|=|\{x \beta: x \in D\}| \leq \aleph_{0} .
$$


Next, we show that $\alpha$ is almost determined by its rational points. For $x \in \mathbb{R} \backslash \mathbb{Q}$ define $s_{x}=\sup _{q \in \mathbb{Q}}\{q \alpha: q<x\}$ and $t_{x}=\inf _{q \in \mathbb{Q}}\{q \alpha: q>x\}$ and observe that

$$
x \alpha \in\left[s_{x}, t_{x}\right],
$$

since $\alpha$ is an order preserving map. Since $\alpha$ is discontinuous at only countably many points there are only countably many intervals $\left[s_{x}, t_{x}\right]$ which are not singletons. It follows that there are only $2^{\aleph_{0}}$ maps in $\mathcal{O}_{\mathbb{R}}$.

The cardinality of the set $\mathcal{P}$ of all order preserving mappings $\mathbb{Q} \rightarrow \mathbb{R}$ is $2^{\aleph_{0}}$. Since every element $\alpha$ of $\mathcal{O}_{\mathbb{R}}$ is almost determined by $\left.\alpha\right|_{\mathbb{Q}} \in \mathcal{P}$ it follows that $\left|\mathcal{O}_{\mathbb{R}}\right|=2^{\aleph_{0}}$ too.

We conclude the paper with the following two questions:

Open Problem 4.13. Is it true that if $\operatorname{rank}\left(\mathcal{T}_{X}: \mathcal{O}_{X}\right) \leq 2$ for a linearly ordered set $X$ then there exists $Y \subseteq X$ such that $|X|=|Y|$ and $Y$, or $Y^{R}$ (the set $Y$ with the order reversed), is well-ordered?

Open Problem 4.14. Does there exist an infinite linearly ordered set $X$ such that $\operatorname{rank}\left(\mathcal{T}_{X}: \mathcal{O}_{X}\right)=2 ?$

ACKNOWLEDGEMENTS. The authors are grateful to an anonymous referee for his/her comments. The second and third authors would also like to thank Dr Lars Olsen for helpful discussions. The third author acknowledges partial support from INTAS 99/1224.

\section{REFERENCES}

1. S. Banach, Sur un théorème de M. Sierpiński, Fund. Math. 25 (1935), 5-6.

2. J. D. Dixon, P. M. Neumann and S. Thomas, Subgroups of small index in infinite symmetric groups, Bull. London Math. Soc. 18 (1986), 560-586.

3. F. Galvin, Generating countable sets of permutations, J. London Math Soc. (2) 51 (1995), 230-242.

4. G. M. S. Gomes and J. M. Howie, On the ranks of certain semigroups of order-preserving transformations, Semigroup Forum 45 (1992), 272-282. 1974).

5. P. R. Halmos, Naive set theory, Undergraduate Texts in Mathematics (Springer-Verlag,

6. P. M. Higgins, Techniques of semigroup theory (The Clarendon Press, Oxford University Press, 1992).

7. P. M. Higgins, J. M. Howie and N. Ruškuc, Generators and factorisations of transformation semigroups, Proc. Roy. Soc. Edinburgh Sect. A 128 (1998), 1355-1369.

8. P. M. Higgins, J. M. Howie, J. D. Mitchell and N. Ruškuc, Countable versus uncountable ranks in infinite semigroups of transformations and relations, Proc. Edinburgh Math. Soc. (2), to appear.

9. J. M. Howie, Products of idempotents in certain semigroups of transformations, Proc. Edinburgh Math. Soc. (2) 17 (1971), 223-236.

10. J. M. Howie, Fundamentals of semigroup theory, London Mathematical Society, New Series No 12 (The Clarendon Press, Oxford University Press, 1995).

11. J. M. Howie, N. Ruškuc and P. M. Higgins, On relative ranks of full transformation semigroups, Comm. Algebra 26 (1998), 733-748.

12. T. J. Jech, Set theory (Academic Press, 1978).

13. K. Kuratowski and A. Mostowski, Set theory (North-Holland Publishing Co., 1968).

14. H. D. MacPherson and P. M. Neumann, Subgroups of infinite symmetric groups, J. London Math. Soc. (2) 42 (1990), 64-84.

15. W. Sierpiński, Sur les suites infinies de fonctions définies dans les ensembles quelconques, Fund. Math. 24 (1935), 209-212. 